\title{
Parathyroid Carcinoma pNO TNM Finding v8
}

National Cancer Institute

\section{Source}

National Cancer Institute. Parathyroid Carcinoma pNO TNM Finding v8. NCI Thesaurus.

Code C141070.

No regional lymph node metastasis. (from AJCC 8th Ed.) 